American Journal of Applied Sciences 4 (11): 880-886, 2007

ISSN 1546-9239

(C) 2007 Science Publications

\title{
Numerical Simulation of 2_D Turbidity Currents and Wall Jet
}

\author{
M.H. Shojaeefard, K. Goudarzi and H. Ghasvari Jahromi \\ Department of Thermofluid and Aerospace Engineering, School of Mechanical Engineering \\ Iran University of Science and Technology, Narmak, 16844, Tehran, Iran
}

\begin{abstract}
Dense underflows are continuous currents, which move down the slope due to the fact that, their density are heavier than ambient water. In turbidity currents the density differences arises from suspended solids. Vicinity of the wall make density currents and wall jets similar in some sense but Variation of density cause this flows more complex than wall jets. An improved form of 'near-wall' k$\varepsilon$ turbulence model is chosen which preserve all characteristics of both density and wall jet currents and a compression is made between them. Then the outcomes from low Reynolds number k- $\varepsilon$ model is compared with $\overline{v^{2}}-f$ model which show similarity. Also results show good agreement with experimental data.
\end{abstract}

Key words: Turbidity currents, wall jet, k- $\varepsilon$ turbulence model, $\overline{v^{2}}-f$ turbulence model

\section{INTRODUCTION}

The gravity currents on the inclined boundaries are formed when the inflow fluid has a density difference with the ambient fluid and a tangential component of gravity becomes the driving force. The salinity concentration and/or the temperature difference cause the density difference. Sometimes, the extra weight of suspend solids causes the density difference, for example turbidity currents in the ocean or in the large lake and powder snow avalanches in the mountains. Turbulent wall jets are an important test case for "general" turbulence models because they contain a near wall as well as a free layer, both interacting with each other. Vicinity of the wall make density currents and wall jets similar in some sense but Variation of density, dynamic instability due to shear forces at the interface of dense layer and ambient fluid with buoyancy forces effect cause this flows more complex than wall jets.

The k- $\varepsilon$ model of turbulence is one of the most extensively used methods but it should be developed to exert damping of lateral velocity fluctuations due to the wall to predict the normal spreading of the wall jets truly. Commonly used eddy viscosity models do not account for the damping of lateral fluctuations by the presence of a wall (unless this is put in empirically) and thus tend to over predict the spreading of the wall jet when empirical constants are used that give the correct spreading for the free jet ${ }^{[1]}$. For example, the widely adopted $\mathrm{k}-\varepsilon$ model $^{[2]}$ produces a spreading rate more than 30 percent too high when the standard constants are used which are suitable for many other flows. One solution for this problem is using Reynolds stress equation models which are complex and expensive in application. However, wall damping effect in wall jets can also be obtained with a simpler model in which the stress equations are reduced to algebraic expression ${ }^{[1]}$. Furthermore density currents are flows, which become unstable and turbulent in low Reynolds number. Hence choosing the low Reynolds number $\mathrm{k}-\varepsilon$ model is necessary due to predominant effect of molecular viscosity on the flow structures in the immediate neighborhood of the wall ${ }^{[3]}$. There are researches on modeling turbulent laden density current such as ${ }^{[4,5]}$ with high Reynolds number k- $\varepsilon$ model. But this model couldn't enter all the characteristics of the density current flows.

Jones and Launder ${ }^{[6]}$ were the first to extend the original k- $\varepsilon$ model to the low -Reynolds-number form which allowed calculations right up to a solid wall. Later, improvements of the $\mathrm{k}-\varepsilon$ model are made successively for the same purpose $\mathrm{e}^{[7-10]}$.

The objective of the present study is to simulate the flow characteristics of 2D turbulent particle laden density current with low Reynolds number k- $\varepsilon$ model $^{[10]}$. Then a compression is made between turbidity current and wall jets in order to specify how

Corresponding Author: $\quad$ K. Goudarzi, Department of Thermofluid and Aerospace Engineering, School of Mechanical Engineering, Iran University of Science and Technology, Narmak, 16844, Tehran, Iran, Tel: +982173913963, Fax: +98 2177491024 
Am. J. Applied Sci., 4 (11): 880-886, 2007

the density differences effect on the structure of turbidity currents. Also the results from low Reynolds number $\mathrm{k}-\varepsilon$ model is compared with $\overline{v^{2}}-f$ model ${ }^{[11]}$ ,that solve $\overline{v^{2}}$ transport equation due to enter damping of lateral velocity fluctuations, which have similarity with Herrero ${ }^{[10]}$. Also results show good agreement with experimental data.

\section{Nomenclature}

$C=\Delta$

$\mathrm{E}$

$h_{0}$

$h$

$g$

$g$,

$P$

$\operatorname{Re}$

$R_{t}=\operatorname{Re}_{t}=\frac{k^{2}}{v \tilde{\varepsilon}} \quad$ turbulence Reynolds number

$\operatorname{Re}_{k}=\frac{y \sqrt{k}}{v} \quad$ dimensionless distance

$\operatorname{Re}_{\tau}=\frac{y u^{*}}{v}=y^{+}$dimensionless distance

$\mathrm{u}^{*}=\sqrt{\frac{\tau_{\omega}}{\rho}} \quad$ friction velocity

$\tau_{\omega}=\left.\mu \frac{\partial \mathrm{u}}{\partial \mathrm{y}}\right|_{\mathrm{y}=0} \quad$ shear stress at wall

$\mathrm{u}_{\mathrm{i}}, \mathrm{u}^{\prime}, \mathrm{v}^{\prime} \quad$ fluctuating velocity in $x_{i}$ direction

$\mathrm{U}_{\text {in }}$

$U_{\text {ave }}$

$U_{\max }$

$x$

$y$

$y_{\max }$

$\mathrm{y}_{1 / 2}$

\section{Greek symbols}

$\lambda$

$\rho$

$\rho_{w}$

$\rho_{s}$

$\theta$

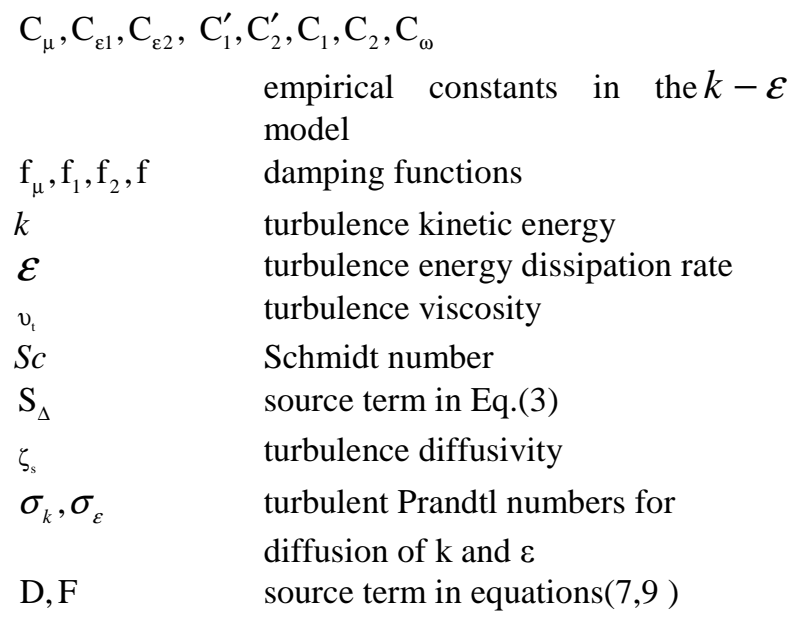

Governing equation: Figure 1 shows the schematic sketch of turbidity current. In the present model computations are based on steady state turbulent turbidity current and wall jet. The concentration of turbid-water is so small that Bosinesque approximation can be used. With this assumption, the effects of density difference are neglected in the inertial term, but included in buoyancy force term in momentum equations. However, the equations of this current are

$\frac{\partial \mathrm{U}_{\mathrm{i}}}{\partial \mathrm{x}_{\mathrm{i}}}=0$

$\mathrm{U}_{\mathrm{j}} \frac{\partial \mathrm{U}_{\mathrm{i}}}{\partial \mathrm{x}_{\mathrm{j}}}=-\frac{1}{\rho_{\mathrm{w}}} \frac{\partial \mathrm{P}}{\partial \mathrm{x}_{\mathrm{i}}}+\frac{\partial}{\partial \mathrm{x}_{\mathrm{j}}}\left(\mathrm{v} \frac{\partial \mathrm{U}_{\mathrm{i}}}{\partial \mathrm{x}_{\mathrm{j}}}-\overline{\mathrm{u}_{\mathrm{i}} \mathrm{u}_{\mathrm{j}}}\right)+\mathrm{g}_{\mathrm{i}} \frac{\rho-\rho_{\mathrm{w}}}{\rho_{\mathrm{w}}}$

$\mathrm{U}_{\mathrm{i}} \frac{\partial \Delta}{\partial \mathrm{x}_{\mathrm{i}}}=\frac{\partial}{\partial \mathrm{x}_{\mathrm{i}}}\left(\lambda+\xi_{\mathrm{s}}\right) \frac{\partial \Delta}{\partial \mathrm{x}_{\mathrm{i}}}+\mathrm{S}_{\Delta}$

Where these equations are, continuity, momentum and mass balances. $\Delta$ is the concentration of fluid defined as $\Delta=\left(\rho-\rho_{\mathrm{w}}\right) /\left(\rho_{\mathrm{s}}-\rho_{\mathrm{w}}\right)$ and $\rho$ is the density of the mixture. $\rho_{s}$ and $\rho_{w}$ are the particles and water density. $v$ and $\lambda$ are the viscosity and diffusivity of fluid, respectively. In the momentum equation, $\mathrm{g}^{\prime}$ is the reduced gravitational acceleration

$g^{\prime}=g \frac{\rho-\rho_{w}}{\rho_{w}}$

In the concentration equation $S_{\Delta}=v_{f} \cos \theta \frac{\partial \Delta}{\partial y}$ is a source term which is added due to particles falling and $v_{f}$ is the particles fall velocity which takes equal to .0014 $\mathrm{m} / \mathrm{s}$ for 50 micron diameter size and $\zeta_{s}$ is the turbulence diffusivity. By using the turbulent Schmidt number $S c$, eddy diffusivity 
$\zeta_{s}=\frac{v_{t}}{S c}$

While the Schmidt number, similar to the Prandtl number is predictable to be affected by the buoyancy, it is assumed to be unity here. The particles in the current are assumed dilute and non-cohesive with equal settling velocities. Pressure term, p, denotes instantaneous pressure which is subtracted from the hydrostatic pressure.

The wall jet governing equation is continuity (1) and momentum equation which expressed as

$U_{j} \frac{\partial U_{i}}{\partial x_{j}}=-\frac{1}{\rho_{w}} \frac{\partial P}{\partial x_{i}}+\frac{\partial}{\partial x_{j}}\left(v \frac{\partial U_{i}}{\partial x_{j}}-\overline{u_{i} u_{j}}\right)+g_{i}$

in the above equation $\mathrm{P}$ is the instantaneous pressure.

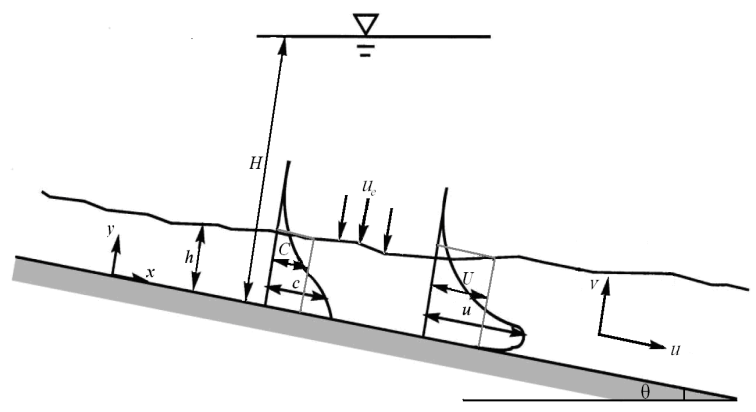

Fig. 1: The schematic sketch of turbidity current

Turbulence modeling: A rigid boundary has many different effects on turbulence ${ }^{[12]}$, the most important of which are as follows :(1) It reduces the length scale of the fluctuations, thus increasing the dissipation rate. (2) It reflects pressure fluctuations, thereby inhibiting the transfer of turbulence energy into fluctuations normal to the wall. (3) It enforces a no-slip condition, thus ensuring that within a wall-adjacent sub-layer, turbulent stresses are negligible and viscous effects on transport processes become of vital importance. Accounting to the second case will lead to correct prediction of normal spreading of wall jet. To note this problem, Ljuboja and Rodi $^{[1]}$ have proposed a modified version of the $\mathrm{k}-\varepsilon$ model. The turbulence scalar quantities ( $k$ and $\varepsilon$ ) used to calculate $v_{t}$ are determined from the following modeled transport equations ${ }^{[10]}$, here $\frac{\partial \mathrm{k}}{\partial \mathrm{t}}=\frac{\partial \tilde{\varepsilon}}{\partial \mathrm{t}}=0$ due to steady solution.

$$
\begin{aligned}
& \frac{\partial k}{\partial t}+U_{i} \frac{\partial k}{\partial x_{i}}=\frac{\partial}{\partial x_{i}}\left[\frac{v_{t}}{\sigma_{k}} \frac{\partial k}{\partial x_{i}}\right]-v_{t}\left(\frac{\partial U_{i}}{\partial x_{j}}+\frac{\partial U_{j}}{\partial x_{i}}\right) \frac{\partial U_{i}}{\partial x_{j}}-\left(1-\frac{\rho_{s}}{\rho_{w}}\right) g_{i} \frac{v_{t}}{\sigma_{t}} \frac{\partial \Delta}{\partial x_{i}} \\
& -\widetilde{\varepsilon}+\mathrm{D}
\end{aligned}
$$

$\frac{\partial \tilde{\varepsilon}}{\partial t}+U_{i} \frac{\partial \tilde{\varepsilon}}{\partial x_{i}}=\frac{\partial}{\partial x_{i}}\left(\frac{v_{t}}{\sigma_{\varepsilon}} \frac{\partial \tilde{\varepsilon}}{\partial x_{i}}\right)-C_{\varepsilon} f_{1} \frac{\tilde{\varepsilon}}{k} v_{t}\left(\frac{\partial U_{i}}{\partial x_{j}}+\frac{\partial U_{j}}{\partial x_{i}}\right) \frac{\partial U_{i}}{\partial x_{j}}-C_{1 \varepsilon} C_{3 \varepsilon} \frac{\tilde{\varepsilon}}{k}\left(1-\frac{\rho_{s}}{\rho_{w}}\right)$

$g_{i} \frac{v_{t}}{\sigma_{t}} \frac{\partial \Delta}{\partial x_{i}}-C_{\varepsilon 2} f_{2} \frac{\tilde{\varepsilon}^{2}}{k}+F$

$\widetilde{\varepsilon}=\varepsilon-\mathrm{D}$

$\sigma_{K}=1, \sigma_{t}=1, \sigma_{\varepsilon}=1.3, C_{\varepsilon 1}=1.44, C_{\varepsilon 2}=1.92 \quad$ are the constants in standard $\mathrm{k}-\varepsilon$ model. Also, the proposed value for $\mathrm{C}_{3 \varepsilon}$ is $.03^{[4]}$.

The reason for including the extra term $\mathrm{D}$ in equation(7) is computational rather than physical; for, in solving the $\varepsilon$ equation, there are decisive advantages, in letting $\varepsilon$ go to zero at the wall. However, the turbulence dissipation rate is not zero there; it is in fact equal to

$\varepsilon_{w}=\left[\overline{\left(\frac{\partial u^{\prime}}{\partial y}\right)^{2}}+\overline{\left(\frac{\partial v^{\prime}}{\partial y}\right)^{2}}\right]$

So, introducing the extra term in equation (7) which is equal to the dissipation rate in the immediate vicinity of the surface and which is negligible in regions where the Reynolds number is high. Also, the last term in the equation (8) is one that found necessary to include in order that the distribution of kinetic energy within the viscosity -affected region should be in reasonable accord with experiment ${ }^{[6]}$.

The Reynolds stress $\overline{u_{i} u_{j}}$ appearing in the system of Eqs.(2,6) may be expressed as:

$-\overline{u_{i} u_{j}}=v_{t}\left(\frac{\partial U_{i}}{\partial x_{j}}+\frac{\partial U_{j}}{\partial x_{i}}\right)-\frac{2}{3} \delta_{i j} k$

The eddy viscosity $v_{t}$ is related to k and $\tilde{\varepsilon}$ through the Kolmogorov-Prandtl relations as:

$v_{t}=C_{\mu} \frac{k^{2}}{\tilde{\varepsilon}}$

For the wall jets considered here, the Reynolds stresses $\overline{u v}, \bar{v}$ of are prime importance and modeling transport equations for these stresses are necessary, but these equations are differential because of the appearance of differential expression in the convective and diffusive transport term, an approximation has been made to reduce these equations to algebraic expressions. Finally, by simplifying differential equations of $\overline{u v}, \overline{v^{2}}$ the $C_{\mu}$ function has been proposed which accounts for the damping effect of the 
wall on the lateral fluctuations for high Reynolds wall jet problems as follow ${ }^{[1]}$ :

$$
\begin{aligned}
\mathrm{C}_{\mu}= & \mathrm{F}_{\mu} \mathrm{G}_{1} \mathrm{G}_{2} \\
\mathrm{G}_{1}= & \frac{1+\frac{3}{2} \frac{\mathrm{C}_{2} \mathrm{C}_{2}^{\prime} \mathrm{f}}{1-\mathrm{C}_{2}}}{1+\frac{3}{2} \frac{\mathrm{C}_{1}^{\prime}}{\mathrm{C}_{1}} \mathrm{f}} \\
\mathrm{G}_{2}= & \frac{1-2 \frac{\mathrm{C}_{2} \mathrm{C}_{2}^{\prime} \mathrm{P} / \widetilde{\varepsilon}}{\mathrm{C}_{1}-1+\mathrm{C}_{2} \mathrm{P} / \tilde{\varepsilon}} \mathrm{f}}{1+2 \frac{\mathrm{C}_{1}^{\prime}}{\mathrm{C}_{1}+\mathrm{P} / \widetilde{\varepsilon}-1} \mathrm{f}} \\
\mathrm{F}_{\mu}= & \frac{2}{3} \frac{1-\mathrm{C}_{2}}{\mathrm{C}_{1}} \frac{\mathrm{C}_{1}-1+\mathrm{C}_{2} \mathrm{P} / \widetilde{\varepsilon}}{\mathrm{C}_{1}+\mathrm{P} / \widetilde{\varepsilon}-1} \\
C_{1}= & 1.8, C_{2}=.6, C_{1}^{\prime}=.6, C_{2}^{\prime}=.3, C_{w}=3.72 \text { are }
\end{aligned}
$$

empirical constants.

In order to impose the effect of low Reynolds in turbidity currents, a version of low-Reynolds-k- $\varepsilon$-model should be chosen. Here, Herrero-model ${ }^{[10]}$ is chosen which contains the effect of the turbulence Reynolds number $R_{t}$, due to predominant effect of molecular viscosity near the wall, and the nondimensional distance from the wall $R_{k}$, due to damping velocity fluctuations in direction normal to the wall, in damping function $f_{\mu}$. In addition, ${ }^{[13]}$ concludes that Herrero mode $^{[10]}$ is more effective to determine the characteristics of wall jets rather than ${ }^{[3]}$ and ${ }^{[9]}$. The damping functions and source terms in this model ${ }^{[10]}$ are as follows:

$$
\begin{aligned}
& f_{\mu}=\left[1-\exp \left(-.006 R_{K}\right)\right]^{2} *\left[1+500 \exp \left(-.005 \mathrm{Re}_{k}\right) / \mathrm{Re}_{t}\right] \\
& f_{1}=1+\left(.05 / f_{\mu}\right)^{2} \\
& f_{2}=1-(.3 / B) \exp \left(-\mathrm{Re}_{t}{ }^{2}\right) \\
& B=1-.7 \exp \left(-\mathrm{Re}_{k}\right) \\
& \mathrm{D}=0 \\
& \mathrm{~F}=0 \\
& \tilde{\varepsilon}_{w}=\frac{\partial \varepsilon}{\partial y}=0
\end{aligned}
$$

Boundary condition: The boundary conditions at the inlet are known. It is similar to the experimental models ${ }^{[14,15]}$, the turbidity current with uniform velocity and concentration enters the channel with $6 \mathrm{~m}$ length and $1 / 1 \mathrm{~m}$ height under the still bodies of water via a sluice gate of $3 \mathrm{~cm}$ height, onto a surface inclined at angle $\theta$.
At the outflow-boundary, the stream wise gradients of all variables are set to zero. At the free surface, the symmetry condition is applied that includes zero gradients and zero fluxes perpendicular to the boundary. At the rigid wall, due to the no slip conditions and a pure depositing assumption, $u_{i}$ and gradient of concentration are set zero. Also for $k-\varepsilon$ equations, at free surface, no flux conditions are imposed, i.e. $\frac{\partial k}{\partial y}=\frac{\partial \varepsilon}{\partial y}=0.0$ and at the inlet $k_{\text {in }}=\left(0.1 u_{\text {in }}\right)^{2}$ and $\widetilde{\mathcal{E}}_{\text {in }}=.0016 u_{\text {in }}{ }^{3} / h_{0}$.

Solution procedure: The flow and the turbulent equations have to be accurately resolved to obtain concentration distribution predictions. All computations were performed in Cartesian coordinates with rectangular geometry. Cartesian grids were used, with a high resolution near all solid boundaries. In all cases, the first grid point was at $\mathrm{y}^{+} \approx 1$ or less. Therefore, the solutions presented here are considered grid independent.

Solver: The governing equations are discreted by a finite-volume method. The continuity, momentum, turbulence and particles mass balance equations are solved in the fixed Cartesian directions on a nonstaggered grid. All the variables are thus stored at the center of the control volume. The velocity components at the control volume faces are computed by the RhieChow interpolation method ${ }^{[16]}$ and the pressure-velocity coupling is handled by SIMPLEC method. The convective terms are treated by the hybrid scheme. TDMA-based algorithms are applied for solving the algebraic equations. The solution procedure is iterative and the computations are terminated when the sums of absolute residuals normalized by the inflow fluxes were below $10^{-4}$ for all variables.

\section{RESULTS AND DISCUSSION}

The height of the dense layer in laboratory is identified by the naked eye of its dim. Therefore, it should be a good opinion to define the height of the dense layer by using the boundary layer concept. Here, we assumed that the interface is a location where its concentration becomes about $1 \%$ of inlet concentration which denotes by $\Delta_{i n}$. Figure 2 show the height of steady density current in comparison with experimental data $^{[14]}$ and $\overline{v^{2}}-f$ model. 
Am. J. Applied Sci., 4 (11): 880-886, 2007

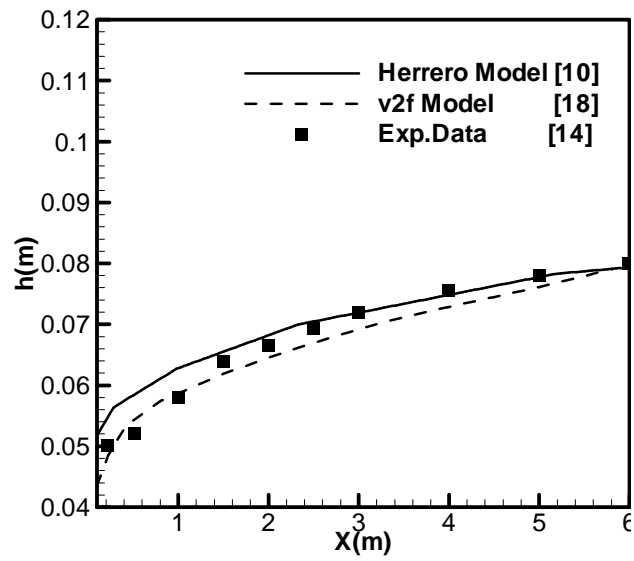

Fig. 2: Height of the turbidity current simulated by Herrero model ${ }^{[10]}$ compared with experimental data and $\overline{v^{2}}-f$ model

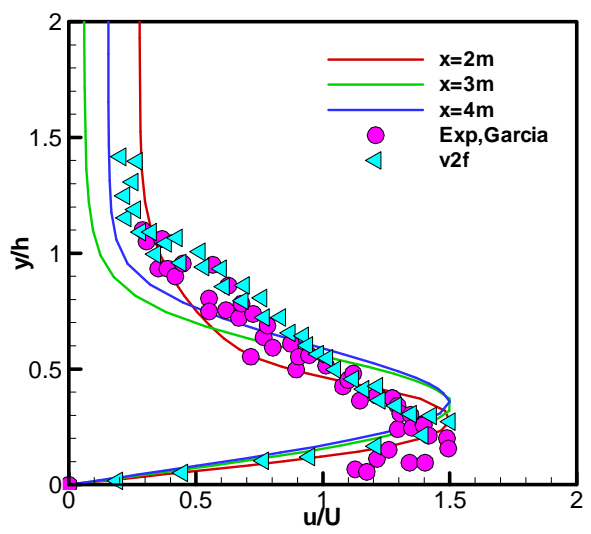

Fig. 3: Velocity profile of the turbidity current along the channel which simulated by Herrero mode ${ }^{[10]}$ compared with experimental data and $\overline{v^{2}}-f$ model

Figure 3 shows velocity profile along the channel, which non-dimensionalized with average velocity versus non -dimensional height of the dense layer, comparing with Experimental data ${ }^{[15]}, u_{i n}=.08 \mathrm{~m} / \mathrm{s}$ as it can be observed the present model simulate the flow properly and the results are similar to $\overline{v^{2}}-f$ model due to the effects of damping function obtained from algebraic equation by simplifying $\overline{v^{2}}$ and $\overline{u v}$ transport equations $^{[1]}$.

Figure 4 shows the horizontal velocity component profile and the along the channel which is simulated by Herrero model ${ }^{[10]}$.

Figure 5 shows the velocity profile of turbidity current and wall jet along the channel. By marching in

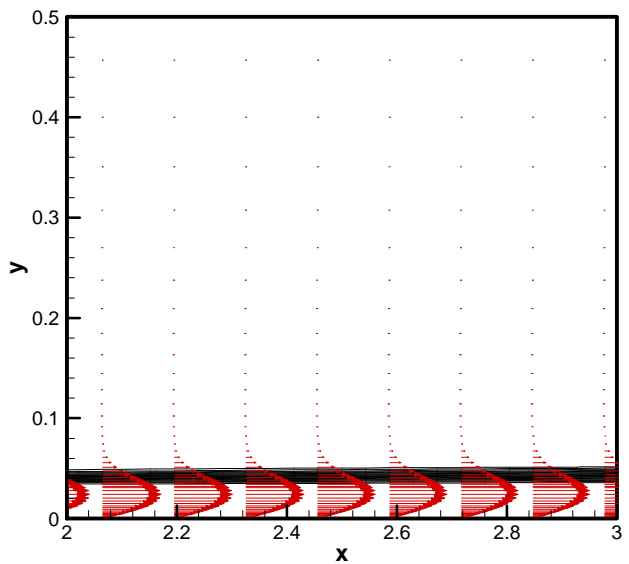

Fig. 4: Horizontal velocity vector and concentration line-isocontours of the turbidity current simulated by Herrero model ${ }^{[10]}$

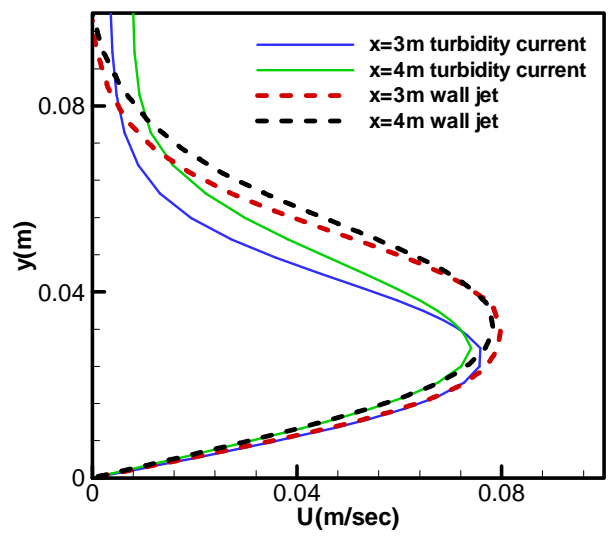

Fig. 5: Comparing velocity profile of wall jet and turbidity current along the channel

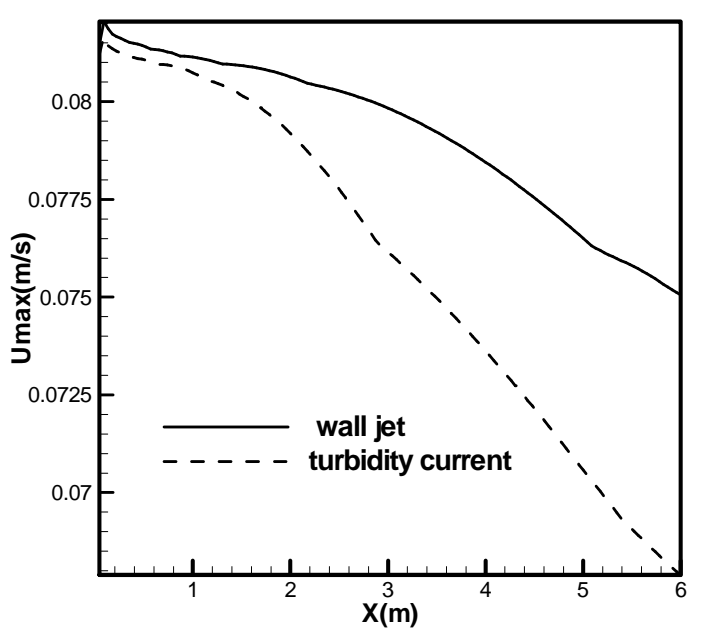

Fig. 6: variation of maximum velocity along the channel for wall jet and turbidity current 


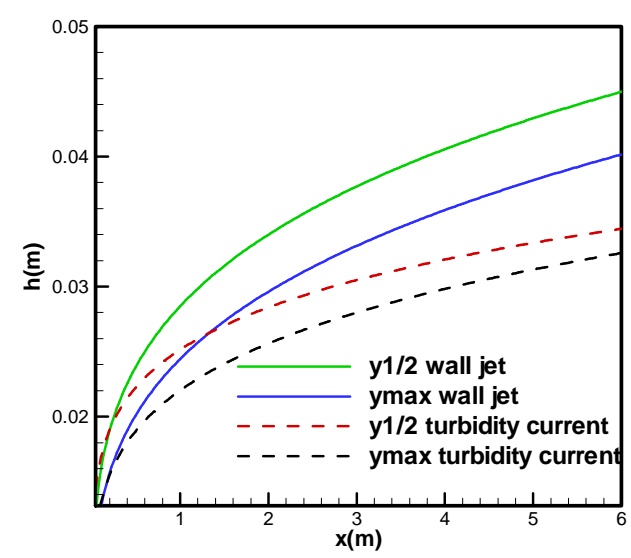

Fig. 7: variation of average velocity along the channel for wall jet and turbidity current

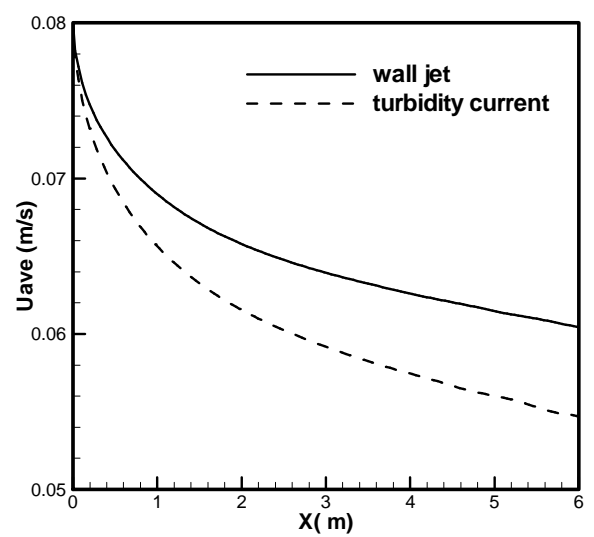

Fig. 8: The heights of turbidity current and wall jet are compared

the stream wise direction the velocity values decrease for both currents and the maximum velocity goes upward due to entrainment. Also the velocity magnitude in wall jet is higher than density current at the same height which leads to higher shear rate and more entrainment for wall jet. It may be as a cause of buoyancy term which represents an exchange between the turbulent kinetic energy $\mathrm{k}$ and potential energy.

Figure 6 and 7 show the variation of maximum velocity and average velocity, respectively, along the channel for both wall jet and turbidity current which is in consistent with Fig. 4.

Average velocity is calculated from bellow equation which is the ratio of momentum to flux at each stream wise cross section ${ }^{[17]}$ :

$$
U_{\text {ave }}=\frac{\int_{0}^{h} U^{2} d y}{\int_{0}^{h} U d y}
$$

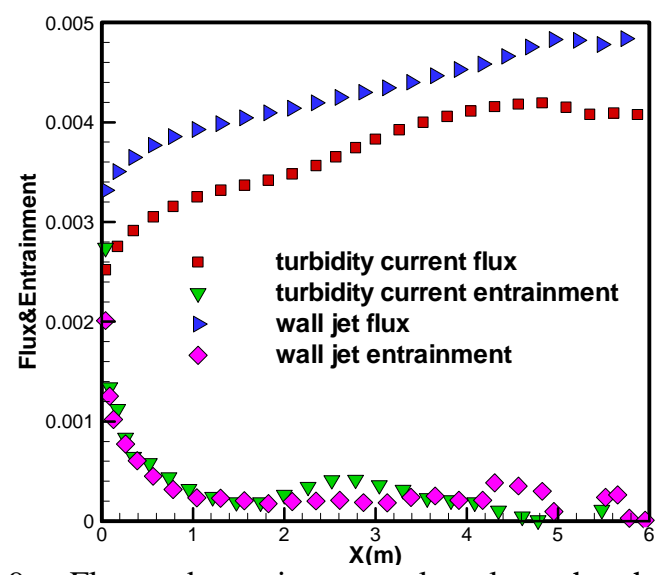

Fig. 9: Flux and entrainment value along the channel for both wall jet and turbidity current

In order to compare the height of turbidity current and wall jet the depth of maximum velocity $y_{\max }$ and $\mathrm{y}_{\frac{1}{2}}{ }^{[18]}$, which is the heights above the maximum velocity where the velocity equals half of the maximum velocity, are calculated (Fig. 8). As it can be observed at the inlet of the channel the heights are the same for both wall jet and turbidity current but after propagation in the stream wise direction the height of the wall jet becomes larger due to higher amount of entraining water.

Due to shear layer at the interface of turbidity current or wall jet and ambient fluid, it disturbs and entrains the surrounding fluid. Turbulence at this boundary entrains the stationary ambient fluid immediately above it, into the layer and dilutes it. The turbulent region grows with distance downstream as the non-turbulent fluid becomes entrained in it. Therefore, a small mean velocity perpendicular to the mean flow is generated when the ambient fluid is initially at rest. The value of Entraining ambient fluid can be obtained from differentiating the flux at each cross section:

$\frac{\mathrm{dUA}}{\mathrm{dx}}=$ Water entrainment

Figure 9 shows the value of flux and entrainment along the channel for both wall jet and turbidity current. Entrainment raises the mass flux and increases the current's height. In wall jet due to high amount of entrained fluid, the velocity profiles move upward and then, its maximum is higher than density current.

\section{CONCLUSION}

The low Reynolds number k- $\varepsilon$ model (Herrero) ${ }^{[10]}$ has been applied to simulate the structure of turbidity 
current. Momentums, continuity, mass balance of particles and turbulence equations are solved, simultaneously, by SIMPLEC method, without any limited or simplify assumption. The computed height of dense layer and velocity profile fall well whit the experimental data. Moreover, results have been compared with the $\overline{v^{2}}-\mathrm{f}$ turbulent model. It has been shown that the results are similar to $\overline{v^{2}}-f$ model due to the effects of damping function obtained from algebraic equation by simplifying $\overline{v^{2}}$ and $\overline{u v}$ transport equations $^{[1]}$. Also a compression is made between the characteristics of wall jet and density currents. It seems that density difference can relax the flow entirely and dissipate some of the flow momentum in both directions. The maximum velocity and height of the wall jet is higher than turbidity current as a result of more amount of entraining ambient fluid.

\section{REFERENCES}

1. Ljuboja, M. and W. Rodi, 1980. Calculation of turbulent wall jets with an aljebraic Reynolds stress model. J. Fluids Eng., 102: 350-356.

2. Rodi, W., 1980. Turbulence model and their application in hydraulics. International Association For Hydraulic Research, Delft, The Netherlands

3. Nagano, Y. and M. Hishida, 1987. Improved form of the k- $\varepsilon$ model for wall turbulent shear flows. J. Fluids Eng., 109: 156-160.

4. Choi, S. and M. Garcia, 2002. K- $-\varepsilon$ Turbulent modeling of density currents developing tow dimensionally on a slope. J. Hydraulic Eng., 128: 1 .

5. Huang, H. and J. Imran, 2005. Numerical model of turbidity currents with a deforming bottom boundary. J. Hydraulic Eng., 131: 283-193.

6. Jones, W.P. and B.E. Launder, 1973. The calculation of low Reynolds number phenomena with a two equation model of turbulence. Int. J. Heat Mass Transfer, 16: 1119-1130.
7. Karimipanah, T., 1996. Turbulent jets in confined spaces. Center for Built Environment Royal Institute or Technology.

8. Lam, C.K.G. and K. Bremhorst, 1981. A modified form of the $\mathrm{k}-\varepsilon$ model for predicting wall turbulence. J. Fluids Eng., 103: 456-460.

9. Chein, K.Y., 1982. Predictions of channel and boundary-layer flows with a low- Reynoldsnumber turbulence model. AIAAJ, 20: 33-38.

10. Herrero, J., F.X. Grau, J. Grifoll and F. Giralt, 1991. A near wall $k-\varepsilon$ formulation for high prandtl number heat transfer. Int. J. Heat Mass Transfer, 34: 711-721.

11. Durbin, P., 1991. Near-wall turbulence closure without damping functions. Theoretical and Computational Fluid Dynamics, 3: 1-13.

12. Launder, B.E., 1989. Second moment closure: present .... and future? Int. J. Heat and Fluid Flow, 10: 282-300.

13. Kechiche, J., H. Mhiri, G. Lepalec and P. Bournot, 2004. Application of low Reynolds number k- $\varepsilon$ turbulence models to the study of turbulent wall jets. Int. J. Thermal Sci., 43: 201-211.

14. Garcia, M.H., 1993. Hydraulic jumps in sediment driven bottom currents. J. Hydraulic Eng., 119: 1094-1117.

15. Akiyama, J., M. Ura and W. Wang, 1994. Physicalbased numerical model of inclined starting plumes. J. Hydraulic Eng., 120: 1139-1157.

16. Rhie, C.M. and W.L. Chow, 1983. Numerical study of the turbulent flow past an airfoil with trailing edge separation. AIAAJ, 21: 1527-1532.

17. Turner, J.S. and T. Ellison, 1959. Turbulent entrainment in stratified flows. J. Fluid Mech., 6: 423-448.

18. Felix, M., 2004. The significance of single value variables in turbidity currents. J. Hydraul. Res., 42: 323-330. 